\title{
Entretien
}

\section{Bernard Delay, au cœur d'un événement majeur : la création du département Environnement et Développement durable au CNRS}

\section{Propos recueillis par Jean-Paul Billaud et Daniel Terrasson}

\author{
Bernard Delay $^{\mathrm{a}}$, Jean-Paul Billaud ${ }^{\mathrm{b}}$, Daniel Terrasson ${ }^{\mathrm{c}}$ \\ a Directeur scientifique, CNRS, Environnement et Développement durable, 3 rue Michel-Ange, 75794 Paris, France \\ b Sociologue, CNRS, UMR7533 LADYSS, Université Paris 10, bâtiment K, 200 avenue de la République, 92001 Nanterre cedex, \\ France \\ c Agronome, CEMAGREF, Direction scientifique, 50 avenue de Verdun, 33612 Cestas cedex, France
}

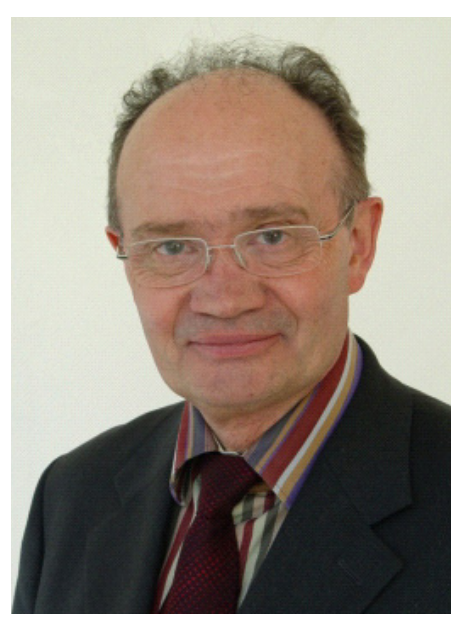

\begin{abstract}
Éléments de biographie
Bernard Delay est né le 29 juillet 1943 à Bedeille (Ariège). Docteur es Sciences de l'université de Lyon (1978).

- Directeur adjoint de l'Institut des sciences de l'évolution (URA CNRS 327), de janvier 1989 à décembre 1995.

- Directeur du département Biologie, évolution, environnement de l'université de Montpellier 2, de juin 1991 à décembre 1995.

- Membre de la Commission de spécialistes (67e section) de l’UM2, jusqu'en 2001.

- Directeur du pôle méditerranéen du programme Environnement du CNRS, jusqu'en juin 2001.

- Directeur de la formation doctorale «Biologie de l'évolution et écologie », UM2 et ENSAM, jusqu'en octobre 2001.

- Membre de la section 30 du comité national (membre du bureau), jusqu'en avril 2001.

- Directeur du centre d'Écologie fonctionnelle et évolutive (CEFE), UPR 9056, CNRS, jusqu'en 2005.

- Directeur scientifique adjoint (DSA) au département des Sciences de la vie (sections 27 et 30) du Comité national de la recherche, jusqu'en 2005.

- Directeur scientifique du département Environnement et Développement durable du CNRS.
\end{abstract}

Jean-Paul Billaud (NSS) : Le CNRS a créé en 2006 le département Environnement et Développement durable (EDD). Cette initiative visait à affronter une question de société particulièrement brûlante et s'accompagnait d'une ambition interdisciplinaire, ce qui représente un événement si l'on se situe dans l'histoire des 30 dernières années de cet établissement. Face à la volonté du département d'informer les communautés scientifiques sur cette création et sur son projet, il nous est apparu que la revue $N S S$ avait vocation à se faire l'écho de ce qu'est EDD.

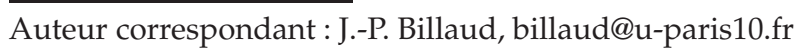

Vous en êtes le directeur, pouvez-vous d'abord nous retracer les conditions de cette création, les motivations qui y ont présidé et la démarche de réflexion qui vous a amené à retenir l'intitulé EDD?

Bernard Delay : Le département EDD est effectivement créé dans l'esprit éditorial de NSS, car il s'agit d'aborder de façon pluridisciplinaire les questions d'environnement et de développement durable. En fait, EDD est issu de deux événements : la création d'un département en janvier 2006, mais aussi la création d'un groupe de disciplines appelé "Sciences de l'environnement ». Ce sont deux événements considérables au 
CNRS. Pourquoi ? Le CNRS s'intéresse depuis longtemps à l'environnement, mais il l'a fait jusqu'à présent sur la base de programmes. La différence entre un département et un programme est importante : le directeur d'un département est à part entière membre du comité de direction du CNRS ; il a donc, sur le plan hiérarchique, exactement le même niveau que les autres directeurs de département, ce qui n'est pas le cas d'un responsable de programme.

Ce département a deux fonctions. La première est celle d'un département classique du CNRS, qui détermine les moyens des unités, suit leur évaluation, conseille leurs directeurs. Aujourd'hui, 68 unités sont rattachées principalement à ce département et, donc, en dépendent directement pour leur évaluation, leurs moyens directs et leur management. Ces unités ont en majorité pour objet la biodiversité (section 29) et le fonctionnement des écosystèmes (les deux tiers de la section 20). Mais 5 unités du domaine $\mathrm{SHS}^{1}$, venant de la section 39 (Espaces, Sociétés, Territoires) et de la section 31 (Hommes et Milieux), ont un rattachement principal au département EDD. Ainsi, EDD a une responsabilité particulière sur les recherches concernant la biodiversité et le fonctionnement des écosystèmes sous influence anthropique variable.

La seconde fonction peut être qualifiée d'agence, à la fois d'objectifs et de moyens. En interne au CNRS, tout ce qui touche à l'environnement et au développement durable le concerne, en interdépartements, donc en interdisciplinaire. Mais il a également pour fonction de dialoguer avec les autres organismes, bien sûr avec les universités - cela est habituel au CNRS -, mais aussi avec toutes les structures impliquées dans le développement durable, que ce soit la Fondation pour le développement durable, l'association $4 \mathrm{D}^{2}, \mathrm{l}^{\prime} \mathrm{IDDRI}^{3}$. N'ayant personne pour le représenter, le CNRS, bien qu'invité, n'y était pas suffisamment présent avant la création d'EDD.

J.-P.B. : Vous parlez d'un événement majeur, est-ce à dire qu'un cap a été franchi par rapport à ce qu'on faisait auparavant, et dans quelles circonstances décisionnelles l'a-t-il été?

Bernard Delay : Il faut se souvenir que le CNRS avait commencé à mettre en place ce qu'on appelait l'Institut national des sciences de l'univers et de l'environnement (INSUE), qui n'était peut-être pas la solution optimale. Au printemps 2005, la question d'une alternative est étudiée au conseil scientifique du CNRS. C'est de là que sont venues la proposition puis la décision, par vote, de créer un département qui s'appellerait Environnement et Développement durable, ce qui fut entériné par Bernard Larrouturou, alors directeur général. La nouvelle équipe

\footnotetext{
${ }^{1}$ Sciences humaines et sociales.

${ }^{2}$ Association Dossiers et débats pour le développement durable.

${ }^{3}$ Institut pour le développement durable et les relations internationales : association loi de 1901 à l'interface de la recherche et de la décision.
}

de direction, autour de Madame Bréchignac, a confirmé et même amplifié cette orientation, en disant clairement que l'environnement et le développement durable étaient une priorité, si ce n'est la priorité pour le CNRS. Par son caractère généraliste, le CNRS, certes pas seul, a tous les éléments pour mettre en œuvre la pluridisciplinarité qu'exige un tel domaine de recherche.

Daniel Terrasson (NSS) : Je voudrais revenir sur la question de 1'INSUE. J'aimerais que vous nous expliquiez en quoi l'INSUE n'était pas en mesure de répondre à cette ambition, parce que, finalement, les mots-clés que vous donnez étaient aussi dans le projet de l'INSUE.

Bernard Delay: On a constaté que l'INSUE n'était pas une solution satisfaisante pour une raison fort simple : c'était un mélange entre les fonctions de l'INSU, qui est un institut national, donc forcément interorganismes, et une fonction interne au CNRS, qui est de développer les recherches sur l'environnement. L'INSUE était un hybride et, de ce fait, ne pouvait pas fonctionner correctement, parce qu'il devait jouer ces deux rôles. Et je me souviens très bien, d'ailleurs, de réunions avec des responsables d'organisme qui nous disaient qu'avec l'INSUE, le CNRS prétendait régner sur tous les aspects des recherches sur l'environnement et sur l'ensemble des organismes, ce qu'ils acceptaient difficilement. Il a donc été préférable de revenir à l'INSU, soit un institut national des sciences de l'univers qui s'intéresse à l'environnement - et on souhaite qu'il s'y intéresse de plus en plus -, mais en interorganismes, distinct du CNRS qui, lui, est doté de son propre outil sur l'environnement. Le département EDD travaille en étroite collaboration avec l'INSU, qui assure le secrétariat du comité interorganismes (CIO). Par décret, le directeur de l'INSU reste responsable du département Planète-Univers, qui est l'ancien département des Sciences de l'univers, mais le CNRS est représenté en tant que tel au comité interorganismes par le département EDD, ce qui le met au même niveau que les autres organismes. Ainsi, les choses sont beaucoup plus claires.

J.-P.B. : Vous dites que l'INSUE n'a pu fonctionner en raison de son caractère hybride, mais vous créez une autre forme d'hybridation. Pourquoi rajouter un département et ne pas s'en tenir à la fonction d'agence interne?

Bernard Delay : Le comité de direction du CNRS est composé, statutairement, des responsables des départements. Donc, si on voulait que l'environnement soit pris en compte à part entière, il fallait faire un département. Un programme n'a pas la plénitude d'un département. À l'origine, le département devait réunir 110 unités, identifiées dans les autres départements, selon qu'elles travaillaient sur l'environnement et de façon pluridisciplinaire. Ce faisant, on voulait aller beaucoup trop vite, car il y avait deux sources d'incompréhension pour les responsables des départements qui considéraient qu'on leur volait leurs unités, et bien souvent parmi leurs meilleures. De leur côté, les unités qui travaillaient sur 
l'environnement ne comprenaient pas très bien pourquoi on les enlevait de leur département d'origine, pour une opération institutionnelle dont l'avenir était incertain. C'était début 2006.

Il a donc semblé beaucoup plus raisonnable de partir sur la base du volontariat. C'est-à-dire que les unités qui souhaitaient être rattachées principalement à EDD pouvaient l'être. Il s'est trouvé que l'ensemble des unités de la section 29, ciblées sur la biodiversité, et les deux tiers des unités de la section 20, ciblées sur le fonctionnement des écosystèmes, ont souhaité être rattachées à EDD. Certains disent qu'il y a eu un effet «Bernard Delay », puisque j'étais le directeur scientifique adjoint (DSA) de ces unités. Je crois que c'est plus profond que cela. Ces unités étaient un peu marginales dans le département des Sciences de la vie, alors qu'avec leur rattachement à EDD, elles se sont positionnées au cœur d'un département dédié à l'environnement. Le fait que j'aie été leur ancien DSA n'est pas anodin, mais l'essentiel était que ces unités voulaient vraiment entrer dans une démarche novatrice. On peut dire la même chose pour les 5 unités qui venaient du département SHS.

Aujourd'hui, d'autres unités voudraient bien bénéficier d'un rattachement principal au département EDD, parce qu'elles constatent que ce n'est pas seulement un effet de mode sans lendemain. Les directeurs d'unité prennent des risques scientifiques, mais n'aiment pas prendre trop de risques institutionnels. Et je les comprends un peu, même si, lorsque j'étais directeur d'unité, je n'étais pas comme cela. Mais on peut les comprendre.

En raison de sa fonction d'agence en interne, ce département a effectivement un statut hybride, mais il n'a fait que la moitié du chemin en tant que département transversal, puisque les unités rattachées à titre principal ne viennent pas de l'ensemble des départements. En revanche, 196 unités lui sont rattachées de façon secondaire. Ce sont des unités qui ont une équipe ou deux travaillant dans le domaine de l'environnement, qui n'avaient aucune raison d'être rattachées principalement - et, d'ailleurs, je ne les aurais pas prises -, mais qui souhaitaient avoir un lien avec EDD pour participer à son animation scientifique.

J.-P.B. : Vous parliez d'une marginalité affectant certaines unités. Pouvez-vous être plus précis?

Bernard Delay : Pour les unités venant de SHS, je crois que ce n'est pas la volonté de sortir d'une marginalité, mais l'envie d'aller vers du nouveau. Concernant les Sciences de la vie, la marginalité tenait au domaine de la biodiversité, qui, à une époque, a même été allégrement piétiné au CNRS. Des directeurs scientifiques n'ont pas hésité à dire leur désintérêt et, d'ailleurs, ils n'y mettaient pas de moyens. Plus récemment, avec Jacqueline Godet, Bernard Pau, Christian Devaux et Michel Vanderesse, l'intérêt de la biodiversité a été affirmé et des moyens spécifiques ont été attribués par le département des Sciences de la vie. Mais elle ne pouvait pas être centrale dans ce département et, de surcroît, elle était en partie à cheval sur le domaine des Sciences de l'univers, pour ce qui relève du fonctionnement des écosystèmes. Avec EDD, le domaine de la biodiversité a trouvé un bol d'air dans un département qui lui est dédié.

J.-P.B. : Ces unités travaillant sur la biodiversité ne se différenciaient-elles pas par des pratiques interdisciplinaires plus importantes?

Bernard Delay: Non, ce n'était pas ça, elles ont trouvé un bol d'air.

D.T. : Vous parlez d'un département «biodiversité » alors que, dans les mots-clés d'EDD, il y a le développement durable. Quel était le projet initial et quel rapport faites-vous entre biodiversité et développement durable?

Bernard Delay : Ce département se définit clairement par son intitulé : Environnement et Développement durable. Du fait des rattachements privilégiant les unités centrées sur la biodiversité, ce département a la responsabilité particulière de cette question. Mais c'est un effet secondaire, car, si beaucoup plus d'unités impliquées dans d'autres problématiques, la chimie par exemple, avaient demandé leur rattachement, le centre de gravité aurait bougé.

Pour bien comprendre la dynamique actuelle, il me faut expliquer ce que le CNRS désigne par «groupe de disciplines ». Les groupes de disciplines réunissent sur certains critères un ensemble de laboratoires. Ils peuvent correspondre à un seul département, comme dans le cas des SHS et de la Chimie, alors qu'en Sciences de la vie, il y en a trois. Suite à la création d'EDD, a été mis en place le groupe de disciplines appelé «Sciences de l'environnement ». Ce groupe de disciplines concerne toutes les unités qui, en juillet 2005, ont affiché $30 \%$ et plus d'activité dans le domaine de l'environnement.

Le département EDD est l'opérateur de ce groupe de disciplines pour l'ensemble des départements, à savoir l'animateur scientifique et le responsable de la répartition des moyens et de la prospective. Il agit donc nécessairement de façon transversale avec les autres départements pour les unités concernées, dont plus de la moitié est hors du département EDD. Ces unités restent dans leur département d'origine, mais, par leur appartenance au groupe de disciplines "Sciences de l'environnement », elles sont parties prenantes de la dynamique scientifique autour de l'environnement et du développement durable.

L'appellation retenue pour le département a été proposée par le conseil scientifique du CNRS au printemps 2005. Mais, avec Catherine Bréchignac, nous nous sommes demandés si «Sciences de l'environnement » ne serait pas plus approprié. Des discussions internes et externes ont eu lieu et nous sommes arrivés à la conclusion que le développement durable, en tant que vraie question de société, appelait des connaissances fondamentales et 
qu'il devait figurer dans l'intitulé du département. La juxtaposition des trois termes apportait un plus par rapport au côté un peu rengaine du terme "environnement».

J.-P.B. : Un tel montage institutionnel basé sur les groupes de disciplines, est-ce une première au CNRS ?

Bernard Delay : C'est une première, même si l'idée avait été discutée à l'époque de Bernard Larrouturou, sans avoir été mise en œuvre. Désormais, les moyens sont affectés aux groupes de disciplines et non aux départements. Évidemment, quand le groupe de disciplines correspond à un département, c'est simple. Dans le cas du groupe de disciplines "Sciences de l'environnement», plus de la moitié des unités sont en dehors du département EDD. L'affectation des moyens est donc discutée entre tous les départements, ce qui confère à EDD un rôle d'arbitre et d'opérateur. Ce groupe est très clairement prioritaire. Ainsi, 14 postes supplémentaires ont été pris à la source et affectés à EDD en plus de la priorité normale du domaine. Ces postes ont été discutés et inscrits dans une politique scientifique. Par exemple, sur ces 14 postes, 8 ont été affichés en SHS, avec l'accord de tous, car les chercheurs travaillant sur ces questions sont insuffisamment nombreux et donc sursaturés.

Un groupe de pilotage, comprenant un représentant de chaque département, vient d'être créé pour travailler sur la mise en œuvre de la stratégie du département dans le cadre de celui du CNRS.

J.-P.B. : EDD, c'est donc tout à la fois un petit département et une grande emprise sur la politique du CNRS?

Bernard Delay: C'est une grande emprise sur la politique du CNRS, dans la mesure où je vois ma mission comme une mission d'opérateur : être l'aiguillon des autres départements tout en respectant leur autonomie. Et je crois que c'est ressenti comme cela. Il est vrai que la création de ce département a été très mal vécue au départ. Au sein du CNRS, comme à l'extérieur, beaucoup disaient que le CNRS avait une vocation de recherche fondamentale et que cela ne servirait à rien, car l'environnement est partout et le développement durable, on ne sait pas ce que c'est. Catherine Bréchignac a tenu bon. Les sceptiques avaient oublié que, dans certains domaines, le besoin d'agir induit des recherches fondamentales générales de haut niveau. Dans les Sciences de la vie, par exemple, une grande partie des recherches de biologie fondamentale est inspirée par des questions médicales. Or, ces recherches sont peu faites par les médecins auprès du malade, elles sont faites par des biologistes, médecins ou non, très souvent sur des modèles biologiques non humains. Ceci conduit à une meilleure compréhension des processus biologiques et permet d'améliorer la thérapie. Pour l'environnement, c'est exactement la même chose. De nombreuses questions qui traduisent ce que certains appellent « la demande sociale » présentent un certain nombre de verrous qui relèvent de la recherche fondamentale, et donc en grande partie du CNRS. Il en est ainsi des recherches sur la biodiversité, avec les questions de métapopulations, de diversité génétique minimale, de transferts de gènes, de fonctionnement des écosystèmes, de microbiologie des sols, de rhizosphère, etc. Même des travaux très pointus en biologie, comme les voies de signalisation chez les végétaux, en relèvent.

J.-P.B. : Vous décrivez ici un lien classique entre recherche fondamentale et «demande sociale». Est-ce à dire qu'il y avait une faillite de l'institution à pouvoir traduire en recherche fondamentale des problèmes qui se posent à la société ? En quoi cela révèle-t-il une limite du système précédent?

Bernard Delay : Il n'y avait pas faillite, car les programmes «Environnement» ont eu de bons résultats. L'apport d'EDD et du groupe de disciplines "Sciences de l'environnement » est de pouvoir traiter un certain nombre de problèmes de façon pluridisciplinaire, dans un cadre prioritaire et dans le respect du domaine de chacun des départements. Prenez le cas des SHS, qui, dans les programmes «Environnement», s'estimaient instrumentalisées. Je crois qu'aujourd'hui, on va arriver à leur donner leur pleine place, en raison de la situation d'opérateur $\mathrm{d}^{\prime} \mathrm{EDD}$, qui doit faire en sorte que chacun s'y retrouve et qu'en particulier, le leader de l'opération soit le département le plus pertinent dans le domaine. Le programme Ville aura d'évidence le département SHS comme leader. En revanche, pour les maladies émergentes, le leader sera plutôt le département des Sciences de la vie, mais le département SHS ou le département Chimie auront leur mot à dire. Pour le programme Chimie pour le développement durable, il en est autrement.

J.-P.B. : Qu'est ce qui donne au département sa légitimité pour faire ce travail de facilitateur? L'apport de finances particulières? En quoi est-ce préférable à un programme?

Bernard Delay : Le département n'a pas repris la fonction du programme. Il a une autre fonction qui est plus en amont. Prenons l'exemple du programme Chimie pour le développement durable, dont l'objectif est de mettre à disposition des produits beaucoup plus propres. C'est un programme de chimie, avec un peu d'économie et d'écologie, puisqu'il est important de considérer les coûts induits ainsi que l'impact de la dégradation de ces produits dans l'environnement. Le rôle du département EDD est différent de celui du programme habituel, car il propose que les dimensions économiques et écologiques soient beaucoup plus fortes. On peut faire le même raisonnement avec le futur programme sur l'énergie. Le département EDD n'a rien à donner, mais il est opérateur. Par la seule discussion, EDD a obtenu une prise en compte significative de la question des économies d'énergie, que ce soit sous ses aspects technologiques, sociaux, organisationnels ou, bien entendu, économiques.

Un programme l'aurait fait, mais pas tout à fait de la même façon. En tant que département, EDD a beaucoup 
plus de poids dans le système. Il tient sa légitimité du fait que la direction du CNRS a décidé que l'environnement était une priorité et que le département EDD était l'outil de cette priorité. Si une autre gouvernance se met un jour en place et opte pour la suppression d'EDD, il n'y aura donc plus de département EDD. Mais revenir en arrière sera compliqué, car le CNRS a besoin de structures transversales par rapport aux départements, tout en maintenant la logique disciplinaire de ceux-ci. Créer les interfaces entre les départements disciplinaires, en s'appuyant sur le groupe de disciplines «Sciences de l'environnement », est le rôle d'un département comme EDD. J'espère que le projet stratégique actuellement en discussion - le CNRS 2020 - affichera l'objectif de création, au sein du CNRS, d'une communauté «Sciences de l'environnement ».

Ce n'est pas forcément une utopie, si vous prenez l'exemple des astronomes. L'astronomie n'est pas une science, mais les astronomes sont des physiciens, des mathématiciens, des chimistes, des biologistes, réunis par une culture et un intérêt communs autour de l'astronomie. Le CNRS se doit de créer cette communauté des sciences de l'environnement. D'ailleurs, j'ai tendance à dire qu'il faudrait arriver à faire cela en France. Mais commençons par le faire chez nous, après nous verrons. Nous n'en sommes pas encore là.

J.-P.B. : Quelles sont les résistances, d'après vous?

Bernard Delay : La peur de l'inconnu, tout d'abord. Des directeurs d'unité viennent me voir en me disant vouloir être rattachés au département EDD, mais ils craignent que l'appartenance à leur communauté disciplinaire disparaisse derrière l'environnement et que ce soit mal jugé par la commission compétente du Comité national. Certains sont prêts à franchir le pas, comme actuellement certains économistes. Mais il faut être patient et je ne cherche pas à forcer ces évolutions. Il faut d'abord construire, il faut que les esprits s'y mettent, et, un jour, il y aura un déclic. Cette nécessité au niveau du CNRS ne disparaîtra pas du jour au lendemain. Tout le monde est maintenant conscient qu'il y a là un problème, et que le CNRS ne peut pas s'en désintéresser. J'en suis convaincu.

D.T. : Parmi les raisons qui ont conduit à la création du département EDD, vous soulignez la volonté de rapprocher la recherche de la société. Vous nous avez expliqué quel en était le sens dans l'identité du CNRS, mais le CNRS se rapproche ainsi d'autres instituts de recherche plus finalisés. Quelles conséquences cela a-t-il sur les relations avec ces établissements, et cela n'entraîne-t-il pas une homogénéisation des conceptions de la recherche dans ces différentes structures?

Bernard Delay : Marion Guillou avait employé pour l'Inra une formule que je trouve excellente: nous sommes un institut de recherche finalisée, mais nous faisons de la recherche fondamentale chaque fois que nous en avons besoin. J'aurais tendance à renverser la proposition pour le CNRS, en disant que nous sommes un institut de recherche fondamentale, mais qui ne se prive pas de faire du travail finalisé lorsque c'est nécessaire. C'est-à-dire lorsque les autres instituts ne s'impliquent pas ou lorsqu'une logique de collaboration s'impose dans un domaine finalisé. Je crois que le CNRS a une place dans de tels dispositifs. Il n'a pas la même fonction ni la même vocation que le Cemagref. Dans le domaine de l'environnement, il se rapproche parfois un peu de l'Inra sur des thématiques comme la nutrition ou le fonctionnement des agrosystèmes, et nous avons mis en place des unités mixtes. Nous travaillons également de plus en plus avec l'IRD et le Cirad. Vis-à-vis des autres organismes, j'essaie d'être à l'écoute. Souvent, ce qui manque, c'est une bonne communication, parce que chacun gère les urgences sans suffisamment de dialogue.

J.-P.B. : Lors des rencontres avec votre département auxquelles j'ai participé en tant que directeur de laboratoire, j'ai été frappé par une dimension que je qualifierai $\mathrm{d}^{\prime}$ « ingénieriale » ou d'expérimentale dans l'approche EDD : sont évoqués des outils, l'écotron, les plateformes techniques, les observatoires de milieux... Cette dimension ingénierale ne participe-t-elle pas du rapprochement avec les pratiques des autres organismes de recherche?

Bernard Delay : Forcément, dans le domaine de l'environnement et du développement durable, la recherche est inspirée par des questions concrètes. Ceci étant, quand nos équipes travaillent sur les matrices parce que nous voulons étudier la dynamique des cormorans ou des mouettes, c'est d'abord un travail sur les matrices. C'est la même chose dans les travaux sur la théorie des métapopulations mobilisée pour aborder les problèmes d'espèces en voie de disparition, de biologie de la conservation ou d'invasions biologiques. C'est pourquoi je serais peu partisan de faire un institut des sciences de l'environnement. Si on mettait tout dans un chaudron et si on «touillait», je ne suis pas certain qu'à la sortie, la synthèse serait meilleure.

J.-P.B. : Ne prenez-vous pas le risque avec la création d'un département Environnement et Développement durable?

Bernard Delay : Non, car il y a l'évaluation qui prend en compte les publications dans des revues internationales de haut niveau. Vous allez me dire que les autres organismes en font autant, mais le rôle des chercheurs du CNRS n'est pas le même : il ne leur est pas interdit de s'occuper un peu des problèmes d'aménagement, mais ce n'est pas leur rôle premier. Je vais prendre un exemple précis : la revégétalisation des pistes de ski soulève des aspects très concrets, nécessite des essais et soulève même des questions de recherche qui sont le fait du Cemagref. Mais, au-delà, se pose le problème des conséquences des dynamiques sur la microflore ou la microfaune du sol. Les équipes du CNRS ont la capacité pour aborder ces aspects et l'idéal est qu'elles les étudient en relation 
avec les équipes du Cemagref. À Grenoble, j'ai souhaité mettre en place une collaboration entre un laboratoire du CNRS, le LECA, et les équipes du Cemagref. Du fondamental aux aspects plus finalisés, nous avons maintenant un continuum.

D.T. : L'ambition du département de mieux répondre aux demandes de la société influence-t-elle le type de recherche, les modes d'intervention dans le débat public? Quelles répercussions sur les pratiques scientifiques, sur la nature des produits de la recherche et sur l'évaluation?

Bernard Delay : L'influence se fera d'abord sentir sur le choix des thèmes, mais aussi sur le rôle social des chercheurs du CNRS. En particulier, ceux-ci doivent mieux s'investir dans les différents comités scientifiques «biodiversité » qui se mettent en place au niveau régional. Quant aux produits de la recherche, cela n'implique pas que les chercheurs du CNRS se lancent dans des études de faisabilité. La réponse est dans la collaboration entre les organismes qui devront passer des conventions et construire des programmes en commun. C'est pour cette raison que j'ai donné mon accord pour engager le programme Eaux et Territoires en partenariat avec le ministère de 1'Environnement et le Cemagref.

J.-P.B.: Quel type de relations un département comme EDD peut-il entretenir avec l'Agence nationale de la recherche (ANR), qui a également un rôle de programmation et, d'une certaine façon, de regroupement de communautés?

Bernard Delay : Très concrètement, ma proposition serait de mettre en place au niveau de l'ANR une coordination de tout ce qui concerne l'environnement, car cette thématique est actuellement dispersée dans plusieurs départements de l'agence. Ce n'est peut-être pas scandaleux en soi, mais il n'y a pas suffisamment de dialogue entre ces départements, et bien souvent les programmes de l'ANR ne sont ni visibles ni vraiment efficaces sur des questions complexes d'environnement. L'ANR devrait afficher clairement ce qu'elle fait dans ce domaine et avoir une vraie stratégie de programmation pluridisciplinaire.

D.T. : Nous avons évoqué à plusieurs reprises le besoin d'interdisciplinarité. Or, nous en connaissons les difficultés. Quelle mesure allez-vous prendre pour dépasser la simple incantation dont elle est souvent l'objet? Quels moyens mettrez-vous pour progresser vers plus d'interdisciplinarité?

Bernard Delay : La définition fonctionnelle que j'ai de l'interdisciplinarité, et que j'ai appliquée dans mes responsabilités précédentes à Montpellier, c'est que chacun doit être au meilleur de sa discipline, mais avec la culture et le respect indispensables pour comprendre et travailler avec d'autres. Il n'y a pas de spécialiste de l'interdisciplinarité. Il n'y a pas non plus de disciplines mineures. Selon la question posée, il y a fatalement une discipline qui est un peu plus en pointe que les autres parce que les questions sont d'abord dans son domaine. Quant aux moyens, ils viennent, par exemple, des programmes interdisciplinaires du CNRS. À présent, le conseil scientifique du CNRS veille à ce que l'interdisciplinarité soit réelle et, évidemment, le département EDD est impliqué. Par contre, vouloir l'interdisciplinarité, ce n'est pas accepter les seuls projets associant une équipe SHS, comme on a pu le lire. Je cite les SHS parce que c'est la question récurrente, mais cela peut concerner d'autres domaines en situation marginale. En revanche, les appels d'offres peuvent très bien être formulés de façon à laisser place à telle ou telle discipline. Motiver les équipes est le point crucial, et nous avons encore à faire un effort d'explication.

D'autre part, lorsque des unités ont effectivement une démarche pluridisciplinaire, nous nous efforçons de les soutenir grâce à des crédits incitatifs. Nous mettons également des moyens sur les $\mathrm{GDR}^{4}$ et, surtout, nous proposons des postes de chercheurs mais aussi des postes d'ITA, comme pour cette année. Ceux-ci sont forcément disciplinaires, mais nous veillons à les affecter dans les domaines qui vont tendre à l'interdisciplinarité. À titre d'exemple, il faut citer l'investissement sur les aspects sociaux ou socioéconomiques de la biologie de la conservation.

D.T. : L'interdisciplinarité fait-elle l'objet d'une animation scientifique particulière?

Bernard Delay : L'animation scientifique est essentielle et nous avons mis en place les Entretiens de Cargèse $^{5}$, avec un objectif $d^{\prime}$ 'ouverture. Une première session concernait l'ingénierie écologique, la seconde était consacrée au thème "Eau et territoire ». Il faut faire en sorte que les gens se rencontrent, et c'est ce qui m'a permis de construire un dialogue productif avec les SHS, alors que la situation était conflictuelle au début de l'année 2006.

J.-P.B. : Le travail fait depuis l'amont au niveau du conseil scientifique du CNRS, jusqu'à l'animation scientifique, se comprend bien, mais ne risque-t-on pas d'être confronté aux vieilles rengaines, sur la difficulté à publier, sur l'absence de reconnaissance dans les carrières pour une recherche qui sort des catégories fondamentale/appliquée et qui est à l'interface des deux? Avezvous également une réflexion sur le type de recherche et les conséquences en termes d'évaluation?

Bernard Delay : Il faudra regarder ce que fera la nouvelle agence d'évaluation, dont les responsables déclarent s'intéresser à ce qui se passe aux interfaces entre les disciplines. Pour le Comité national, nous faisons passer des messages sur la prise en compte positive de l'interdisciplinarité. Quand nous avons mis en place le conseil scientifique du département EDD, qui est en fait celui du groupe de disciplines «Sciences de

\footnotetext{
${ }^{4}$ Groupement de recherche : une structure légère et fédérative mise en place conjointement par le CNRS et le ministère de la Recherche.

5 Séminaires thématiques de réflexion organisés dans le centre CNRS de Cargèse, en Corse.
} 
l'environnement ", nous avons pris soin que les nominations couvrent tous les domaines. Nous amorçons un système, mais les changements prendront du temps et il faut compter sur les jeunes, qui sont beaucoup plus réceptifs. Chaque chercheur doit trouver le bon équilibre entre des publications dans sa discipline et un certain nombre d'articles plus transversaux. NSS a un rôle à jouer, à condition de se démarquer d'une image trop SHS. Au niveau international, il existe maintenant, dans le champ de l'environnement, de plus en plus de revues qui acceptent des articles multidisciplinaires.

J.-P.B. : Au niveau européen, la démarche EDD estelle unique sur le plan institutionnel?

Bernard Delay : Non. Prenez le Natural Environment Council (NERC) : sa démarche est similaire à celle d'EDD dans un contexte différent. De ce côté-là, je pense même que la France est un peu en retard.

J.-P.B. : Pour finir, nous aimerions savoir ce qui vous a conduit à prendre en main ce projet? En quoi y a-t-il une cohérence entre le poste que vous occupez aujourd'hui, les choix que vous avez faits en termes de politique scientifique et votre parcours personnel?
Bernard Delay : J'occupe ce poste tout à fait par hasard, car je suis venu pour 6 mois début 2006 pour rendre service suite à un désistement : mi-2007, je suis encore là. Quant à mon parcours, j'ai commencé à travailler sur le domaine cavernicole en étudiant la dynamique des populations de coléoptères souterrains, qui était alors totalement incomprise. Ces coléoptères étaient trouvés occasionnellement dans les grottes, mais on ne savait même pas où ils étaient précisément, ce qui m'a amené à analyser de multiples paramètres entrant dans le champ de diverses disciplines. Ensuite, je suis parti à Montpellier, où j'ai eu la chance de travailler avec Louis Taller, qui était quelqu'un d'extrêmement ouvert sur le plan intellectuel. Puis j'ai dirigé le CEFE, qui était déjà interdisciplinaire et que j'ai rendu encore plus interdisciplinaire. Enfin, avant d'être directeur de ce département EDD, j'ai été, pendant 4 ans, directeur adjoint du département des Sciences de la vie, en charge de l'environnement et de la biodiversité. Je crois que c'est un problème de tempérament et de philosophie de la recherche.

To access this journal online: www.edpsciences.org 